\title{
Non-syndromic male infertility due to sperm motility disorder
}

INSERM

\section{Source}

INSERM. (1999). Orphanet: an online rare disease and orphan drug data base. Nonsyndromic male infertility due to sperm motility disorder. ORPHA:276234

Non-syndromic male infertility due to sperm motility disorder is a rare, genetic, nonsyndromic male infertility disorder characterized by infertility due to sperm with defects in their cilia/flagella structure, leading to absent motility or reduced forward motility in fresh ejaculate. Reduced semen volume, oligospermia and an increased number of abnormally structured spermatozoa is often present. 